International Mathematical Forum, 2, 2007, no. 64, 3155 - 3167

\title{
Undesirable Factors in Efficiency Evaluation with Interval Data
}

\author{
F. Hosseinzadeh Lotfi ${ }^{1}$ \\ Department of Mathematics, Science \& Research Branch \\ Islamic Azad University, Tehran, Iran \\ A. A. Noura \\ Department of Mathematics \\ University of Sistan \& Baluchestan, Zahedan, Iran \\ G. R. Jahanshahloo \\ Department of Mathematics \\ Teacher Training University,Tehran, Iran \\ Z. Iravani \\ Department of Mathematics, Science \& Research Branch \\ Islamic Azad University, Tehran, Iran
}

\begin{abstract}
Efficiency measurment is usually based on the assumption that inputs have to be minimized and outputs have to be maximized. Many production processes yield both desirable (inputs/outputs) and undesirable factors. There are some models that evaluate efficiency level in the presence of undesirable factors. The purpose of this paper develop interval efficiency in presence both desirable (inputs/outputs) and undesirable factors.
\end{abstract}

Keywords: Data envelopment analysis; Radial efficiency measurment; Non- radial DEA models; Interval data; Undesirable factory

\footnotetext{
${ }^{1}$ Corresponding author. farhad@hosseinzadeh.ir (Farhad Hosseinzadeh Lotfi)
} 


\section{Introduction}

Data envelopment analysis (DEA) is a non parametric techniqe for measuring and evaluating the relative efficiency of DMUs which stand for decision making units with several inputs and outputs[1,2]. classical DEA models rely on the assumption that inputs have to be minimized and outputs have to maximized.However, it was mentioned already[2] that the production process may also generate undesirable outputs like smoke pollution or waste. In some situations, some inputs need to be increased and some outputs need to be decreased to improve the performance of a DMU.

The aim of this paper is to introduce new DEA models that will allow DEA to be applied using interval data in presence input-output desirable and undesirable factors.

We assume that there are $\mathrm{n}$ DMUs to be evaluated, indexed by $j=1, \ldots, n$ and each DMU is assumed to produce $s$ different outputs from $m$ different inputs. Let the observed input and output vectors of $D M U_{j}$ be $X_{j}=\left(x_{1 j}, \ldots, x_{m j}\right)$ and $Y_{j}=\left(y_{1 j}, \ldots, y_{s j}\right)$ respectively, that all component of vectors $X_{j}$ and $Y_{j}$ for all DMUs are non-negative and each DMU had at least one strictly positive input and output.

following charnes et al.[1] and banker et al.[3] we assume that the technology set is estimated by

$T_{v}=\left\{(X, Y) \mid X \geq \sum_{j=1}^{n} \lambda_{j} X_{j}, Y \leq \sum_{j=1}^{n} \lambda_{j} Y_{j}, \sum_{j=1}^{n} \lambda_{j}=1, \lambda_{j} \geq 0, j=1, \ldots, n\right\}$

Suppose the DEA data domain is expressed as

$$
\left[\begin{array}{c}
Y \\
-X
\end{array}\right]=\left[\begin{array}{c}
Y^{g} \\
Y^{b} \\
-X
\end{array}\right]
$$

Where $Y^{g}$ and $Y^{b}$ represent the desirable(good) and undesirable(bad) output respectively. Obviously we wish to increase the $Y^{g}$ and to decrease the $Y^{b}$ to improve the performance.

Since the possibility condition in presence undesirable factor is

$$
\begin{gathered}
(X, Y) \in T, Y=\left(Y^{g}, Y^{b}\right) \Rightarrow\left(X, Y^{g}, Y^{b}\right) \in T \\
X^{\prime} \geq X \Rightarrow\left(X^{\prime}, Y^{g}, Y^{b}\right) \in T \\
Y^{\prime g} \leq Y^{g} \Rightarrow\left(X, Y^{\prime g}, Y^{b}\right) \in T \\
Y^{\prime b}=Y^{b} \Rightarrow\left(X, Y^{g}, Y^{\prime b}\right) \in T
\end{gathered}
$$


Then the technology sets is estimated by

$$
\begin{gathered}
T_{v}^{\prime}=\left\{\left(X, Y^{g}, Y^{b}\right) \mid \sum_{j=1}^{n} \lambda_{j} X_{j} \leq X, \sum_{j=1}^{n} \lambda_{j} Y_{j}^{g} \geq Y^{g}, \sum_{j=1}^{n} \lambda_{j} Y_{j}^{b}=Y^{b}, \sum_{j=1}^{n} \lambda_{j}=1\right. \\
\left., \lambda_{j} \geq 0, j=1, \ldots, n\right\}
\end{gathered}
$$

Now suppose the input vector is displaced by the $m$ rows vector $V$ and the desirable and undesirable output vectors is displaced by the $s$ rowed vectors $U 1, U 2$. that is $\bar{X}_{j}=X_{j}+V, \bar{Y}_{j}=Y_{j}^{g}+U_{1}, \bar{Y}_{j}^{b}=Y_{j}^{b}+U_{2}, \mathrm{j}=1, \ldots, \mathrm{n}$ Ali and seiford [2] provide the following result concerning the translation in the $B C C$ model. This is approach $[R T R \beta]$. In approach $[T R \beta]$ we multiply each undesirable output by " -1 " and then fined a proper translation vector $\beta$ to let all negative undesirable outputs be positive. The data domain of (1) now becomes

$$
\left[\begin{array}{c}
Y \\
-X
\end{array}\right]=\left[\begin{array}{c}
Y^{g} \\
\bar{Y}^{b} \\
-X
\end{array}\right]
$$

where the jth column of(translated) bad output now is $\bar{y}_{j}^{b}=-y_{j}^{b}+\beta>0$ based upon (2) model $B C C$ becomes the following linear program

$$
\begin{array}{cl}
\max & \phi \\
\text { s.t. } & \sum_{j=1}^{n} \lambda_{j} x_{j} \leq x_{o} \\
& \sum_{j=1}^{n} \lambda_{j} y_{j}^{g} \geq \phi y_{o}^{g} \\
& \sum_{j=1}^{n} \lambda_{j} \bar{y}_{j}^{b}=\phi \bar{y}_{o}^{b} \\
& \sum_{j=1}^{n} \lambda_{j}=1 \\
\lambda_{j} \geq 0, \quad j=1, \ldots, n
\end{array}
$$

Now,consider the data is $: x_{i j} \in\left[x_{i j}^{L}, x_{i j}^{U}\right], y_{r j}^{g} \in\left[y_{r j}^{g L}, y_{r j}^{g U}\right]$ and for undesirable output

$$
\begin{gathered}
y_{r j}^{L} \leq y_{r j} \leq y_{r j}^{U} \Longrightarrow-y_{r j}^{U} \leq-y_{r j} \leq-y_{r j}^{L} \Longrightarrow-y_{r j}^{U}+\beta \leq-y_{r j}+\beta \leq-y_{r j}^{L}+\beta \\
\Longrightarrow \bar{y}_{r j}^{U} \leq \bar{y}_{r j} \leq \bar{y}_{r j}^{L}
\end{gathered}
$$

By this data, modify models (3) the following interval linear programing prob- 
lem:

$$
\begin{array}{ll}
\phi_{o}=\max & \phi \\
\text { s.t. } & \sum_{j=1}^{n} \lambda_{j}\left[x_{j}^{L}, x_{j}^{U}\right] \leq\left[x_{o}^{L}, x_{o}^{U}\right] \\
& \sum_{j=1}^{n} \lambda_{j}\left[y_{j}^{g L}, y_{j}^{g U}\right] \geq \phi\left[y_{o}^{g L}, y_{o}^{g U}\right] \\
& \sum_{j=1}^{n} \lambda_{j}\left[\bar{y}_{j}^{b L}, \bar{y}_{j}^{b U}\right]=\phi\left[\bar{y}_{o}^{b L}, \bar{y}_{o}^{b U}\right] \\
& \sum_{j=1}^{n} \lambda_{j}=1 \\
& \lambda_{j} \geq 0, \quad j=1, \ldots, n
\end{array}
$$

We compute by the following models the upper and lower bounds of the interval of efficiency rating for $D M U O$ :

$$
\begin{aligned}
& \phi_{o}^{L}=\max \phi \\
& \text { s.t. } \quad \sum_{\substack{j=1 \\
j \neq 0}}^{n} \lambda_{j} x_{i j}^{L}+\lambda_{o} x_{i o}^{U} \leq x_{i o}^{U}, \quad i=1, \ldots, m \\
& \sum_{\substack{j=1 \\
j \neq 0}}^{n} \lambda_{j} y_{r j}^{g U}+\lambda_{o} y_{\text {ro }}^{g L} \geq y_{\text {ro }}^{g L}, \quad r=1, \ldots, s \\
& \sum_{\substack{j=1 \\
j \neq 0}}^{n} \lambda_{j} \bar{y}_{r j}^{b L}+\lambda_{o} \bar{y}_{r o}^{b U}=\bar{y}_{r o}^{b U}, \\
& \sum_{j=1}^{n} \lambda_{j}=1 \\
& \lambda_{j} \geq 0, \quad j=1, \ldots, n \\
& \phi_{o}^{U}=\max \quad \phi \\
& \text { s.t. } \quad \sum_{\substack{j=1 \\
j \neq 0}}^{n} \lambda_{j} x_{i j}^{U}+\lambda_{o} x_{i o}^{L} \leq x_{i o}^{L}, \quad i=1, \ldots, m \\
& \sum_{\substack{j=1 \\
j \neq 0}}^{n} \lambda_{j} y_{r j}^{g L}+\lambda_{o} y_{r o}^{g U} \geq y_{r o}^{g U}, \quad r=1, \ldots, s \\
& \sum_{\substack{j=1 \\
j \neq 0}}^{n} \lambda_{j} \bar{y}_{r j}^{b U}+\lambda_{o} \bar{y}_{r o}^{b L}=\bar{y}_{r o}^{b L}, \quad r=1, \ldots, s \\
& \sum_{j=1}^{n} \lambda_{j}=1 \\
& \lambda_{j} \geq 0, \quad j=1, \ldots, n
\end{aligned}
$$

Theorem 1. If $x_{i j} \in\left[x_{i j}^{L}, x_{i j}^{U}\right], y_{r j}^{g} \in\left[y_{r j}^{g L}, y_{r j}^{g U}\right], y_{r j}^{b} \in\left[y_{r j}^{b L}, y_{r j}^{b U}\right], \phi^{*}, \phi_{o}^{L^{*}}, \phi_{o}^{U^{*}}$ are the optimum objective function values of $(3),(5),(6)$ models respectively, then $\phi^{*} \in\left[\phi^{L^{*}}, \phi^{U^{*}}\right]$.

proof. Consider the feasible region for models (3), (5), (6) , $S_{3}, S_{5}, S_{6}$ respectively, we will proof $S_{5} \subseteq S_{3} \subseteq S_{6}$.

Consider the first constraint in models (5)

$$
\sum_{\substack{j=1 \\ j \neq 0}}^{n} \lambda_{j} x_{i j}^{U}+\lambda_{o} x_{i o}^{L} \leq x_{i o}^{L} \Longrightarrow \sum_{\substack{j=1 \\ j \neq 0}}^{n} \lambda_{j} x_{i j}^{U} \leq\left(1-\lambda_{o}\right) x_{i o}^{L}
$$


Since $x_{i o}^{L} \leq x_{i o}$ then by $\sum_{j=1}^{n} \lambda_{j}=1$ we have

$$
\left(1-\lambda_{o}\right) x_{i o}^{L} \leq\left(1-\lambda_{o}\right) x_{i o}
$$

but

$$
x_{i j}^{U} \geq x_{i j} \Longrightarrow \sum_{\substack{j=1 \\ j \neq 0}}^{n} \lambda_{j} x_{i j}^{U} \geq \sum_{\substack{j=1 \\ j \neq 0}}^{n} \lambda_{j} x_{i j}
$$

Now by $\left(1^{\prime}\right),\left(2^{\prime}\right),\left(3^{\prime}\right)$ we have

$$
\sum_{\substack{j=1 \\ j \neq 0}}^{n} \lambda_{j} x_{i j} \leq \sum_{\substack{j=1 \\ j \neq 0}}^{n} \lambda_{j} x_{i j}^{U} \leq\left(1-\lambda_{o}\right) x_{i o}^{L} \leq\left(1-\lambda_{o}\right) x_{i o}
$$

then $\sum_{\substack{j=1 \\ j \neq 0}}^{n} \lambda_{j} x_{i j} \leq\left(1-\lambda_{o}\right) x_{i o}$.

Now consider the second constraint in models(5). In model (5) we have

$$
\sum_{\substack{j=1 \\ j \neq 0}}^{n} \lambda_{j} y_{r j}^{g L}+\lambda_{o} y_{r o}^{g U} \geq \phi y_{r o}^{g U} \Longrightarrow \sum_{\substack{j=1 \\ j \neq 0}}^{n} \lambda_{j} y_{r j}^{g L} \geq\left(\phi-\lambda_{o}\right) y_{r o}^{g U}
$$

since $y_{r o}^{g U} \geq y_{\text {ro }}$ and $\sum_{j=1}^{n} \lambda_{j}=1$ and $\phi \geq 1$ we have

$$
\left(\phi-\lambda_{o}\right) y_{r o}^{g U} \geq\left(\phi-\lambda_{o}\right) y_{\text {ro }}
$$

but

$$
y_{\text {ro }}^{g} L \leq y_{r o} \Longrightarrow \sum_{\substack{j=1 \\ j \neq 0}}^{n} \lambda_{j} y_{r j}^{g L} \leq \sum_{\substack{j=1 \\ j \neq 0}}^{n} \lambda_{j} y_{r j}
$$

Now by $\left(4^{\prime}\right),\left(5^{\prime}\right),\left(6^{\prime}\right)$ we have

$$
\sum_{\substack{j=1 \\ j \neq 0}}^{n} \lambda_{j} y_{r j} \geq \sum_{\substack{j=1 \\ j \neq 0}}^{n} \lambda_{j} y_{r j}^{g L} \geq\left(\phi-\lambda_{o}\right) y_{r o}^{g U} \geq\left(\phi-\lambda_{o}\right) y_{r o}
$$

then $\sum_{\substack{j=1 \\ j \neq 0}}^{n} \lambda_{j} y_{r j} \geq\left(\phi-\lambda_{o}\right) y_{r o}$. The proof of third constraint is similar first and second constrain. The proof is complete.

classification invariance: $D M U O$ is efficient under $(3),(5),(6)$ if and only if $D M U O$ is efficient under (3), (5), (6) with translated data, DMUo is inefficient under (3), (5), (6) if and only if DMUo is inefficient under (3), (5), (6) with translate data.

The models (3), (5), (6) based upon classification invariance. 
The above discussion can also be applied to situation when some inputs need to be increased rather than decreased to improve the performance. In this case, we rewrite the $D E A$ data domain as

$$
\left[\begin{array}{c}
Y \\
-X
\end{array}\right]=\left[\begin{array}{c}
Y \\
-X^{I} \\
-X^{D}
\end{array}\right]
$$

Where $X^{I}$ and $X^{D}$ represent inputs to be increased and decreased, respectively.Next multiply $X^{I}$ by " -1 " and then find a proper translation vector $k$ to let all negative $X^{I}$ be positive. The data domain of (7) becomes

$$
\left[\begin{array}{c}
Y \\
-X
\end{array}\right]=\left[\begin{array}{c}
Y \\
-\bar{X}^{I} \\
-X^{D}
\end{array}\right]
$$

Where the jth column of(translation) input to be increase now is $\bar{x}_{j}^{I}=-x_{j}^{I}+k>0$

The model in input-oriented is similar to output-oriented. The rest of this paper organize as follows:In section (2) we discuss about the non-radial model for efficiency evaluate.In section (3) we discuss interval data in non-radial model. In section (4) and (5) we use those model in presence undesirable factor in interval data.

\section{The non-radial model for efficiency evaluate}

Efficiency measurment is usually based on the assumption that desirable inputs and undesirable outputs have to minimized and desirable outputs and undesirable inputs have to be maximized.

There is consider both of the undesirable factors in non-radial model for efficiency evaluate. This method preserve linearity and convexity.

Consider the additive model in $B C C$

$$
\begin{array}{clr}
h_{o}^{1}=\max & \sum_{i=1}^{n} s_{i}^{-}+\sum_{r=1}^{s} s_{r}^{+} & \\
\text {s.t. } & \sum_{j=1}^{n} \lambda_{j} x_{i j}+s_{i}^{-}=x_{i o} & i=1, \ldots, m \\
& \sum_{j=1}^{n} \lambda_{j} y_{r j}-s_{r}^{+}=y_{r o} & r=1, \ldots, s \\
& \sum_{j=1}^{n} \lambda_{j}=1 & \\
& \lambda_{j} \geq 0, & j=1, \ldots, n \\
& s_{i}^{-} \geq 0, s_{r}^{+} \geq 0, & i=1, \ldots, m, r=1, \ldots, s
\end{array}
$$

since the objective function depended input and output unit we divide input and output to $R_{i}^{-}$and $R_{r}^{+}$,respectively, where $R_{i}^{-}=\max _{1 \leq j \leq n}\left\{x_{i j}\right\}, R_{r}^{+}=$ 
$\max _{1 \leq j \leq n}\left\{y_{r j}\right\}$.

So,the equivalent form of the model (9) is as follows:

$$
\begin{array}{rlc}
h_{o}^{2}=\max & \sum_{i=1}^{n} \frac{s_{i}^{-}}{R_{i}^{-}}+\sum_{r=1}^{s} \frac{s_{r}^{+}}{R_{r}^{+}} & \\
\text {s.t. } & \sum_{j=1}^{n} \lambda_{j} \frac{x_{i j}}{R_{i}^{-}}+\frac{s_{i}^{-}}{R_{i}^{-}}=\frac{x_{i o}}{R_{i}^{-}} & i=1, \ldots, m \\
& \sum_{j=1}^{n} \lambda_{j} \frac{y_{r j}}{R_{r}^{+}}-\frac{s_{r}^{+}}{R_{r}^{+}}=\frac{y_{r o}}{R_{r}^{+}} & r=1, \ldots, s \\
& \sum_{j=1}^{n} \lambda_{j}=1 & \\
& \lambda_{j} \geq 0, & j=1, \ldots, n \\
& s_{i}^{-} \geq 0, s_{r}^{+} \geq 0, & i=1, \ldots, m, r=1, \ldots, s
\end{array}
$$

So,the equivalent form of the model (10) is as follows:

$$
\begin{array}{crr}
h_{o}^{3}=\min \quad 1- & {\left[w_{o}+\frac{1}{m+s}\left(\sum_{i=1}^{m} t_{i}^{-}+\sum_{r=1}^{s} t_{r}^{+}\right)\right]} & \\
\text {s.t. } & \sum_{j=1}^{n} \lambda_{j} x_{i j}+t_{i}^{-}=x_{i o}-w_{o} & i=1, \ldots, m \\
& \sum_{j=1}^{n} \lambda_{j} y_{r j}-t_{r}^{+}=y_{r o}+w_{o} & r=1, \ldots, s \\
& \sum_{j=1}^{n} \lambda_{j}=1 & \\
& t_{i}^{-} \geq 0, t_{r}^{+} \geq 0, & i=1, \ldots, m, r=1, \ldots, s \\
& w_{o}, \lambda_{j} \geq 0, & j=1, \ldots, n
\end{array}
$$

Where $t_{i}^{-}=\frac{s_{i}^{-}}{R_{i}^{-}}-w_{0}, i=1, \ldots, m$ and $t_{r}^{+}=\frac{s_{r}^{+}}{R_{r}^{+}}-w_{o}, r=1, \ldots, s$

Theorem 2. DMUo in model (10) is efficiency if and only if $h_{o}^{3^{*}}=1$

Proof. The proof is straightforward.

\section{Interval data in non-radial model}

Suppose the DEA data domain is expressed as

$$
x_{i j} \in\left[x_{i j}^{L}, x_{i j}^{U}\right], y_{r j}^{g} \in\left[y_{r j}^{g L}, y_{r j}^{g U}\right], y_{r j}^{b} \in\left[y_{r j}^{b L}, y_{r j}^{b U}\right]
$$


So,the model (9) is modified in the following form:

$$
\begin{array}{rlr}
h_{o}=\max & \sum_{i=1}^{n} s_{i}^{-}+\sum_{r=1}^{s} s_{r}^{+} & \\
\text {s.t. } & \sum_{j=1}^{n} \lambda_{j}\left[x_{i j}^{L}, x_{i j}^{U}\right]+s_{i}^{-}=\left[x_{i o}^{L}, x_{i o}^{U}\right] & i=1, \ldots, m \\
& \sum_{j=1}^{n} \lambda_{j}\left[y_{r j}^{g L}, y_{r j}^{g U}\right]-s_{r}^{+}=\left[y_{r o}^{g L}, y_{r o}^{g U}\right] & r=1, \ldots, s \\
& \sum_{j=1}^{n} \lambda_{j}\left[y_{r j}^{b L}, y_{r j}^{b U}\right]-s_{r}^{+}=\left[y_{r o}^{b L}, y_{r o}^{b U}\right] & \\
& \sum_{j=1}^{n} \lambda_{j}=1 & \\
& \lambda_{j} \geq 0, & j=1, \ldots, s \\
& s_{i}^{-} \geq 0, s_{r}^{+} \geq 0, &
\end{array}
$$

Compute by the following models the upper and lower bounds of the interval of efficiency rating for $D M U O$

$$
\begin{aligned}
& h_{o}=\max \quad \sum_{i=1}^{m} s_{i}^{-}+\sum_{r=1}^{s} s_{r}^{+} \\
& \text {s.t. } \quad \sum_{\substack{j=1 \\
j \neq 0}}^{n} \lambda_{j} x_{i j}^{L}+\lambda_{o} x_{i o}^{U}+s_{i}^{-}=x_{i o}^{U} \quad i=1, \ldots, m \\
& \sum_{\substack{j=1 \\
j \neq 0}}^{n} \lambda_{j} y_{r j}^{U}+\lambda_{o} y_{r o}^{L}+s_{r}^{+}=y_{r o}^{L} \quad r=1, \ldots, s \\
& \sum_{j=1}^{n} \lambda_{j}=1 \\
& \lambda_{j} \geq 0 \text {, } \\
& j=1, \ldots, n \\
& s_{i}^{-} \geq 0, s_{r}^{+} \geq 0, \quad i=1, \ldots, m, r=1, \ldots, s \\
& h_{o}=\max \quad \sum_{i=1}^{m} s_{i}^{-}+\sum_{r=1}^{s} s_{r}^{+} \\
& \text {s.t. } \quad \sum_{\substack{j=1 \\
j \neq 0}}^{n} \lambda_{j} x_{i j}^{U}+\lambda_{o} x_{i o}^{L}+s_{i}^{-}=x_{i o}^{L} \quad i=1, \ldots, m \\
& \sum_{\substack{j=1 \\
j \neq 0}}^{n} \lambda_{j} y_{r j}^{L}+\lambda_{o} y_{r o}^{U}+s_{r}^{+}=y_{r o}^{U} \quad r=1, \ldots, s \\
& \sum_{j=1}^{n} \lambda_{j}=1 \\
& \lambda_{j} \geq 0 \\
& j=1, \ldots, n \\
& s_{i}^{-} \geq 0, s_{r}^{+} \geq 0 \text {, } \\
& i=1, \ldots, m, r=1, \ldots, s
\end{aligned}
$$

For normalized inputs and outputs we have $R_{i}^{-}=\max _{1 \leq j \leq n}\left\{x_{i j}^{U}\right\}$, $R_{r}^{+}=\max _{1 \leq j \leq n}\left\{y_{r j}^{U}\right\}$. 
So,the models (14) and (15) as following

$$
\begin{array}{rlr}
h_{o}^{L}=\max & \sum_{i=1}^{n} \frac{s_{i}^{-}}{R_{i}^{-}}+\sum_{r=1}^{s} \frac{s_{r}^{+}}{R_{r}^{+}} & \\
\text {s.t. } & \sum_{\substack{j=1 \\
j \neq 0}}^{n} \lambda_{j} \frac{x_{i j}^{L}}{R_{i}^{-}}+\lambda_{o} \frac{x_{i o}^{U}}{R_{i}^{-}}+\frac{s_{i}^{-}}{R_{i}^{-}}=\frac{x_{i o}^{U}}{R_{i}^{-}} & i=1, \ldots, m \\
& \sum_{\substack{j=1 \\
j \neq 0}}^{n} \lambda_{j} \frac{y_{r j}^{U}}{R_{r}^{+}}+\lambda_{o} \frac{y_{r o}^{L}}{R_{r}^{+}}-\frac{s_{r}^{+}}{R_{r}^{+}}=\frac{y_{r o}^{L}}{R_{r}^{+}} & \\
& \sum_{j=1}^{n} \lambda_{j}=1 & \\
& \lambda_{j} \geq 0, & j=1, \ldots, s \\
& s_{i}^{-} \geq 0, s_{r}^{+} \geq 0, & i=1, \ldots, m, r=1, \ldots, s
\end{array}
$$

$$
\begin{array}{rlr}
h_{o}^{U}=\max & \sum_{i=1}^{n} \frac{s_{i}^{-}}{R_{i}^{-}}+\sum_{r=1}^{s} \frac{s_{r}^{+}}{R_{r}^{+}} & \\
\text {s.t. } & \sum_{\substack{j=1 \\
j \neq 0}}^{n} \lambda_{j} \frac{x_{i j}^{U}}{R_{i}^{-}}+\lambda_{o} \frac{x_{i o}^{L}}{R_{i}^{-}}+\frac{s_{i}^{-}}{R_{i}^{-}}=\frac{x_{i o}^{L}}{R_{i}^{-}} & i=1, \ldots, m \\
& \sum_{\substack{j=1 \\
j \neq 0}}^{n} \lambda_{j} \frac{y_{r j}^{L}}{R_{r}^{+}}+\lambda_{o} \frac{y_{r o}^{U}}{R_{r}^{+}}-\frac{s_{r}^{+}}{R_{r}^{+}}=\frac{y_{r o}^{U}}{R_{r}^{+}} & r=1, \ldots, s \\
& \sum_{j=1}^{n} \lambda_{j}=1 & \\
& \lambda_{j} \geq 0, & \\
& s_{i}^{-} \geq 0, s_{r}^{+} \geq 0, & i=1, \ldots, m, r=1, \ldots, s
\end{array}
$$

The equivalent form of the models (16) and (17) are as follows:

$$
\begin{array}{lll}
h^{L}=\min & 1-\left[w_{o}+\frac{1}{m+s}\left(\sum_{i=1}^{m} t_{i}^{-}+\sum_{r=1}^{s} t_{r}^{+}\right)\right] & \\
\text {s.t. } & \sum_{\substack{j=1 \\
j \neq 0}}^{n} \lambda_{j} x_{i j}^{L}+\lambda_{o} x_{i o}^{U}+t_{i}^{-}=x_{i o}^{U}-w_{o} & i=1, \ldots, m \\
& \sum_{\substack{j=1 \\
j \neq 0}}^{n} \lambda_{j} y_{r j}^{U}+\lambda_{o} y_{r o}^{L}-t_{r}^{+}=y_{r o}^{L}+w_{o} & r=1, \ldots, s \\
& \sum_{j=1}^{n} \lambda_{j}=1 \\
& t_{i}^{-} \geq 0, t_{r}^{+} \geq 0, & i=1, \ldots, m, r=1, \ldots, s \\
& w_{o}, \lambda_{j} \geq 0, & j=1, \ldots, n
\end{array}
$$




$$
\begin{array}{rlr}
h^{U}=\min & 1-\left[w_{o}+\frac{1}{m+s}\left(\sum_{i=1}^{m} t_{i}^{-}+\sum_{r=1}^{s} t_{r}^{+}\right)\right] & \\
\text {s.t. } & \sum_{\substack{j=1 \\
j \neq 0}}^{n} \lambda_{j} x_{i j}^{U}+\lambda_{o} x_{i o}^{L}+t_{i}^{-}=x_{i o}^{L}-w_{o} & i=1, \ldots, m \\
& \sum_{\substack{j=1 \\
j \neq 0}}^{n} \lambda_{j} y_{r j}^{L}+\lambda_{o} y_{r o}^{U}-t_{r}^{+}=y_{r o}^{U}+w_{o} & r=1, \ldots, s \\
& \sum_{j=1}^{n} \lambda_{j}=1 \\
& t_{i}^{-} \geq 0, t_{r}^{+} \geq 0, & \\
& w_{o}, \lambda_{j} \geq 0, & \\
& & j=1, \ldots, m, r=1, \ldots, s \\
& &
\end{array}
$$

Where $\frac{s_{i}^{-}}{R_{i}^{-}}=t_{i}^{-}+w_{o}$ and $\frac{s_{r}^{+}}{R_{r}^{+}}=t_{r}^{+}+w_{o}$.

Theorem 3. DMUO is efficient in models (18) and (19) if and only if $h^{L *}=$ $h^{U *}=1$

\section{The non-radial model for efficiency evaluate in pres- ence undesirable inputs and outputs in interval data}

Suppose the inputs and outputs divide into two categories of desirable and undesirables ones. That is $X_{j}=\left(X_{j}^{D}, X_{j}^{U}\right)$ and $Y_{j}=\left(Y_{j}^{D}, Y_{j}^{U}\right)$ Where $X_{j}^{U}$ and $Y_{j}^{U}$ represent the undesirable inputs and outputs and $X_{j}^{D}$ and $Y_{j}^{D}$ represent the desirable inputs and outputs of $D M U_{j}$, respectively. To improve the performance of $D M U O, X_{o}^{U}$ and $Y_{o}^{D}$ have to be increased and $X_{o}^{D}$ have to be decreased. To do so model (11) is modified in the following form:

$$
\begin{array}{ccc}
h_{o}^{4}=\min & 1-\left[w_{o}+\frac{1}{m+s}\left(\sum_{i=1}^{m} t_{i}^{-D}+\sum_{r=1}^{s} t_{r}^{+D}\right)\right] & \\
\text { s.t. } & \sum_{j=1}^{n} \lambda_{j} x_{i j}^{D}+t_{i}^{-D}=x_{i o}^{D}-w_{o} & i \in I_{D} \\
& \sum_{j=1}^{n} \lambda_{j} x_{i j}^{U}=x_{i o}^{U}-w_{o} & i \in I_{U} \\
& \sum_{j=1}^{n} \lambda_{j} y_{r j}^{D}-t_{r}^{+D}=y_{r o}^{D}+w_{o} & r \in O_{D} \\
& \sum_{j=1}^{n} \lambda_{j} y_{r j}^{U}=y_{r o}^{U}+w_{o} & \\
& \sum_{j=1}^{n} \lambda_{j}=1 & \\
& t_{i}^{-} \geq 0, t_{r}^{+} \geq 0, & i=1, \ldots, m, r=1, \ldots, s \\
& w_{o}, \lambda_{j} \geq 0, & j=1, \ldots, n
\end{array}
$$

Here $I_{D}, O_{D}, I_{U}$ and $O_{D}$ are the indices set for the desirable inputs, desirable outputs, undesirable inputs and undesirable outputs, respectively. In (20) we use the normalized data. DMUo is efficiency in the model (20) if and only if $h_{o}^{4 *}=1$. 
Theorem 4. The model (20) is feasible and bounded.

Theorem 5. If in the optimal solution of $(20) w_{o}^{*}=0$, then at least one of slacks in this solution is zero.

Suppose the inputs and outputs divide into two categories of desirable and undesirable ones in interval data, that is:

$$
X_{j}^{D} \in\left[X_{j}^{D L}, X_{j}^{D U}\right], X_{j}^{U} \in\left[X_{j}^{U L}, X_{j}^{U U}\right], Y_{j}^{D} \in\left[Y_{j}^{D L}, Y_{j}^{D U}\right], Y_{j}^{U} \in\left[Y_{j}^{U L}, Y_{j}^{U U}\right]
$$

Where $X_{j}^{U}$ and $Y_{j}^{U}$ represent the undesirable inputs and outputs and $X_{j}^{D}$ and $Y_{J}^{D}$ represent the desirable inputs and outputs of $D M U j$,respectively.

To improve the performance of $D M U O, X_{o}^{U}$ and $Y_{o}^{D}$ have to be increased and $X_{o}^{D}$ and $Y_{o}^{U}$ have to decreased. To do so, models (18) and (19) are modified in the following form:

$$
\begin{aligned}
& {h^{\prime}}^{L}=\min \quad 1-\left[w_{o}+\frac{1}{m+s}\left(\sum_{i \in I_{D}} t_{i}^{-}+\sum_{r \in O_{D}} t_{r}^{+}\right)\right] \\
& \text {s.t. } \quad \sum_{\substack{j=1 \\
j \neq 0}}^{n} \lambda_{j} x_{i j}^{D L}+\lambda_{o} x_{i o}^{D U}+t_{i}^{-D}=x_{i o}^{D U}-w_{o} \quad i \in I_{D} \\
& \sum_{\substack{j=1 \\
j \neq 0}}^{n} \lambda_{j} x_{i j}^{U U}+\lambda_{o} x_{i o}^{U L}=x_{i o}^{U L}-w_{o} \quad i \in I_{U} \\
& \sum_{\substack{j=1 \\
j \neq 0}}^{n} \lambda_{j} y_{r j}^{D U}+\lambda_{o} y_{r o}^{D L}-t_{r}^{+D}=y_{r o}^{D L}+w_{o} \quad r \in O_{D} \\
& \sum_{\substack{j=1 \\
j \neq 0}}^{n} \lambda_{j} y_{r j}^{U L}+\lambda_{o} y_{r o}^{U U}=y_{r o}^{U U}+w_{o} \quad r \in O_{U} \\
& \sum_{j=1}^{n} \lambda_{j}=1 \\
& t_{i}^{-} \geq 0, t_{r}^{+} \geq 0, \quad i=1, \ldots, m, r=1, \ldots, s \\
& w_{o}, \lambda_{j} \geq 0, \quad j=1, \ldots, n
\end{aligned}
$$




$$
\begin{array}{ccc}
h^{\prime \prime U}=\min & 1-\left[w_{o}+\frac{1}{m+s}\left(\sum_{i \in I_{D}} t_{i}^{-}+\sum_{r \in O_{D}} t_{r}^{+}\right)\right] & \\
\text {s.t. } & \sum_{\substack{j=1 \\
j \neq 0}}^{n} \lambda_{j} x_{i j}^{D U}+\lambda_{o} x_{i o}^{D L}+t_{i}^{-D}=x_{i o}^{D L}-w_{o} & i \in I_{D} \\
& \sum_{\substack{j=1 \\
j \neq 0}}^{n} \lambda_{j} x_{i j}^{U L}+\lambda_{o} x_{i o}^{U U}=x_{i o}^{U U}-w_{o} & i \in I_{U} \\
& \sum_{\substack{j=1 \\
j \neq 0}}^{n} \lambda_{j} y_{r j}^{D L}+\lambda_{o} y_{r o}^{D U}-t_{r}^{+D}=y_{r o}^{D U}+w_{o} & r \in O_{D} \\
& \sum_{\substack{j=1 \\
j \neq 0}}^{n} \lambda_{j} y_{r j}^{U U}+\lambda_{o} y_{r o}^{U L}=y_{r o}^{U L}+w_{o} & \\
& \sum_{j=1}^{n} \lambda_{j}=1 \\
& t_{i}^{-} \geq 0, t_{r}^{+} \geq 0, & \\
& w_{o}, \lambda_{j} \geq 0, & j=1, \ldots, m=1, \ldots, s \\
& j=1, \ldots, n
\end{array}
$$

Theorem 6. Suppose we have data in $(21)$ then $h^{*} \in\left[h^{\prime L *}, h^{\prime \prime}{ }^{*}\right]$

Proof.The proof is straightforward.

Theorem 7. If $h^{L *}=h^{\prime \prime U *}=1$ then $D M U O$ is efficient.

Theorem 8. The models (22) and (23) are feasible and bounded.

Theorem 9. The models (22) and (23) are unit invariant.

\section{6.conclusion}

Efficiency measurment is usually based on the assumption that inputs have to be minimized and outputs have to be maximized. In a growing number of application, however, undesirable outputs(inputs)are incorporated into the production model which have to be minimized(maximized). In this paper we investigated the non-radial model in presence undesirable factors in interval data. As a result, convexity and linearity are preserved. we demonastred the proposed model is feasible and bounded.

\section{References}

[1] Charnes A., W. W. Cooper and E. Rhodes, " Measuring the efficient of decision making unit", European Journal of Operation Research 2(1978) 429-444.

[2] Ali, A. I., L. M Seiford, " Translation invariance in data envelopment analysis", Operation Research Letters 9(1990),403-405.

[3] Charnes A., W.W. Cooper, B. Golany, L.Seiford and J.stutz " Foundation of data envelopment analysis for Pareto-Koopmans efficient empirical production function", Journal of Econometrice 30(1985)91-107.

[4] Tone k., " measurment and Improvement of efficiency by DEA", Nikkagiren publishers, 1993(In Japanese) 
[5] Banker R. D., A.Charnes and W. W. Cooper, " some methods for estimating technical and scale inefficiency in data envelopment analysis", Management science 30(9)(1984)1078-1092.

[6] Koopmans T.C. " analysis of production as an efficient combination activities", T.C. Koopmans (Ed.)Activity Analysis of production and Allocation, Cowles commission, Wilely New York, (1951)33-97.

Received: June 20, 2007 